\title{
Title: Acute effects of self-myofascial release compared to dry needling on myofascial pain syndrome related outcomes: range of motion, muscle soreness and performance. A randomized controlled trial.
}

J Vicente-Mampel

Catholic University of Valencia

\section{IJ Bautista}

Catholic University of Valencia

\section{J López-Soler}

Catholic University of Valencia

J Torregrosa-Valls

Catholic University of Valencia

P Gargallo ( $D$ pedro.gargallo@ucv.es )

Catholic University of Valencia

\section{Baraja-Vegas}

Catholic University of Valencia

\section{Research Article}

Keywords: foam roller, countermovement jump, hyperalgesia, range of motion, myofascial trigger point pain

Posted Date: October 7th, 2021

DOI: https://doi.org/10.21203/rs.3.rs-941900/v1

License: (c) (i) This work is licensed under a Creative Commons Attribution 4.0 International License.

Read Full License 
Title: Acute effects of self-myofascial release compared to dry needling on myofascial pain syndrome related outcomes: range of motion, muscle soreness and performance. A randomized controlled trial.

Head title: Effects of foam roller vs dry needling on myofascial pain.

Vicente-Mampel, $\mathrm{J}^{1}$, Bautista $\mathrm{IJ}^{1}$, López-Soler $\mathrm{J}^{1}$, Torregrosa-Valls $\mathrm{J}$, Gargallo $\mathrm{P}^{1^{*}}$, Baraja-Vegas $L^{1}$

${ }^{1}$ Department of Physiotherapy, Faculty of Medicine and Health Science. Catholic University of Valencia, Torrent, Valencia, Spain.

Corresponding author: Department of Physiotherapy, Catholic University of Valencia, Torrent, Valencia, Spain. e-mail: pedro.gargallo@ucv.es, phone: +34 656660506 


\begin{abstract}
Objectives: myofascial pain syndrome (MPS) is associated with reductions in range of movement (ROM), decrease in physical function and performance and increase in pain in different populations. Elucidating the best prevention and treatment strategies for MPS has been one of the main goals in the last decade. The objective of the present study was to compare the acute effects of self-myofascial foam rolling (SFR) and dry-needling (DN) techniques on ankle dorsiflexion ROM, soreness post-needling and performance through countermovement jump (CMJ) height.
\end{abstract}

Methods: a prospective crossover design composed by 12 active adults, aged $23.41 \pm$ 1.68 years (weight: $78.33 \pm 9.02 \mathrm{Kg}$; height: $1.79 \pm 0.088 \mathrm{~m}$ ) with active ankle dorsiflexion restriction was performed. Participants were randomized into the SFR and DN techniques to analyze its effects on ankle dorsiflexion ROM, muscle soreness and jump height at pre, post and $24 \mathrm{~h}$ post-intervention.

Results: significant improvements were found by DN on ankle dorsiflexion at 24 hours post intervention and muscle soreness by SFR at the same time point. No significant differences were found between conditions.

Conclusions: Both SFR and DN are effective in improving ankle dorsiflexion and performance in young adults without producing an acute effect in muscle soreness.

Keywords: foam roller, countermovement jump, hyperalgesia, range of motion, myofascial trigger point pain. 


\section{INTRODUCTION}

Injury prevention and recovery strategies are two basic areas in sport and physical exercise fields. One of the aspect that have generated most interest in the last decade in these two areas has been the myofascial pain syndrome (MPS) ${ }^{1}$. MPS usually appears when the demands in sport and exercise activities exceeds the tolerance capacity of the muscle tissue, leading with pain, weakness and lack of mobility ${ }^{2}$. The appearance of myofascial trigger points (MTrPs) has been described within the MPS as something common ${ }^{3}$.These are defined as a hypersensitive area in a tight band of a muscle ${ }^{4}$.

In recent years, foam rolling has become one of the main tools used for the treatment of MPS as well as for the recovery of muscle tissue after the damage produced by the sport and exercise activities 5 . Foam rolling has shown beneficial effects on improving hamstring range of movement (ROM), muscle stiffness, late onset of pain and also pressure pain threshold (PPT) ${ }^{6}$.

On the other hand, another treatment used in MPS is the dry needling (DN) technique ${ }^{7}$. This tool has been shown to be effective to relieve pain and increase ROM in the shortterm ${ }^{8}$. For example, a meta-analysis performed by Gattie, Cleland and Snodgrass 9 revealed that very low quality to moderate quality evidence suggests that $\mathrm{DN}$ is more effective that no treatment for reducing pain and improving PPT.

Interestingly, a recent meta-analysis leaded by Wiewelhove et al. ${ }^{10}$ showed that a treatment with SFR shows minors and partially negligible positive effects on performance and muscle recovery. However, the use of foam roller could be beneficial in some cases 
as it increases sprint performance and flexibility. In addition, previous studies have detected that self-myofascial foam roller (SFR) can also reduce muscle pain sensation ${ }^{11}$.

However, regarding the DN technique, one of the main side-effects is the appearance of post-needling soreness. This post-needling soreness response is variable, and despite the fact that not all subjects experience it with the same intensity level, the risk of the its appearance could determine the choice of this technique over others ${ }^{12}$. However, this symptom does not have to affect the muscle contractile properties ${ }^{13}$.

Therefore, the present study aimed to examine and compare the acute effects in terms of magnitude and duration of SFR and DN techniques on ankle mobility, pain and jump height in active and healthy young adults. We hypothesized that both interventions, SFR and DN, would lead similar acute (immediate and post 24h) effects on all the parameters analyzed, with no significant differences between conditions in any of them. 


\section{RESULTS}

Table 1 shows the descriptive statistics of all dependent variables analyzed during both intervention conditions. The 12 participants who where initially randomly assigned to each condition received the treatment and were analyzed for the primary and secondary variables. There were no dropouts.

\begin{tabular}{|c|c|c|c|c|}
\hline Variable & $\begin{array}{c}\text { Self-myofascial } \\
\text { foam roller }\end{array}$ & Dry needling & $\begin{array}{c}\text { Mean Difference } \\
{\left[\mathrm{CI}_{95 \%}\right]}\end{array}$ & $\%$ of change \\
\hline \multicolumn{5}{|c|}{ Ankle dorsiflexion ROM } \\
\hline Pre & $27.92(3.50)$ & $27.33(4.07)$ & $0.58[-3.21$ to 4.38$]$ & 2 \\
\hline Immediate Post & $29.83(3.71)$ & $29.33(3.62)$ & $0.50[-3.30$ to 4.30$]$ & 2 \\
\hline Post $24 \mathrm{~h}$ & $28.00(3.51)$ & $26.41(3.17)$ & $1.58[-2.21$ to 5.38$]$ & 6 \\
\hline \multicolumn{5}{|c|}{ Post intervention soreness (EVA) } \\
\hline Pre & $3.37(1.38)$ & $3.39(1.26)$ & $-0.02[-1.73$ to 1.70$]$ & -1 \\
\hline Immediate Post & $3.23(1.41)$ & $3.19(1.18)$ & $0.04[-1.68$ to 1.76$]$ & -1 \\
\hline Post $24 \mathrm{~h}$ & $2.30(1.32)$ & $3.38(1.67)$ & $-1.08[-2.80$ to 0.63$]$ & -47 \\
\hline \multicolumn{5}{|c|}{ CMJ height (cm) } \\
\hline Pre & $30.77(4.53)$ & $31.58(4.69)$ & $-0.81[-4.68$ to 3.06$]$ & -3 \\
\hline Immediate Post & $31.69(5.02)$ & $31.34(4.69)$ & $0.35[-3.52$ to 4.22$]$ & 1 \\
\hline Post $24 \mathrm{~h}$ & $33.43(5.67)$ & $31.34(7.30)$ & $2.09[-1.78$ to 5.96$]$ & 6 \\
\hline
\end{tabular}

Note $=$ all variables met the normality assumption $(\mathrm{p}>0.05), \mathrm{CMJ}=$ countermovement jump, ROM= range of motion.

$\mathrm{CI}_{95 \%}=$ confidence interval at $95 \%$.

Table 1. Effects of intervention. 
The RM ANOVA did not show significant differences in main effect of "intervention" variable $\left(\mathrm{F}_{[1,11]}=1.39, \mathrm{p}=0.264\right.$, eta $\left.=0.05\right)$ on lunge test. The $\mathrm{MD}, \mathrm{CI}_{95 \%}$ and $\mathrm{ES}$ were 0.89 (-0.77 to 2.55$)$ and 0.34 , respectively. On the other hand, non-significant differences were found at interaction effect of "time $x$ intervention" $\left(F_{[2,22]}=0.28, p=0.759, \mathrm{n}^{2} \mathrm{p}=\right.$ 0.02). Bonferroni post hoc comparison revealed significant differences in post immediate intervention vs. post 24 hours post intervention in DN condition (see figure 1).

\section{Experimental conditions}

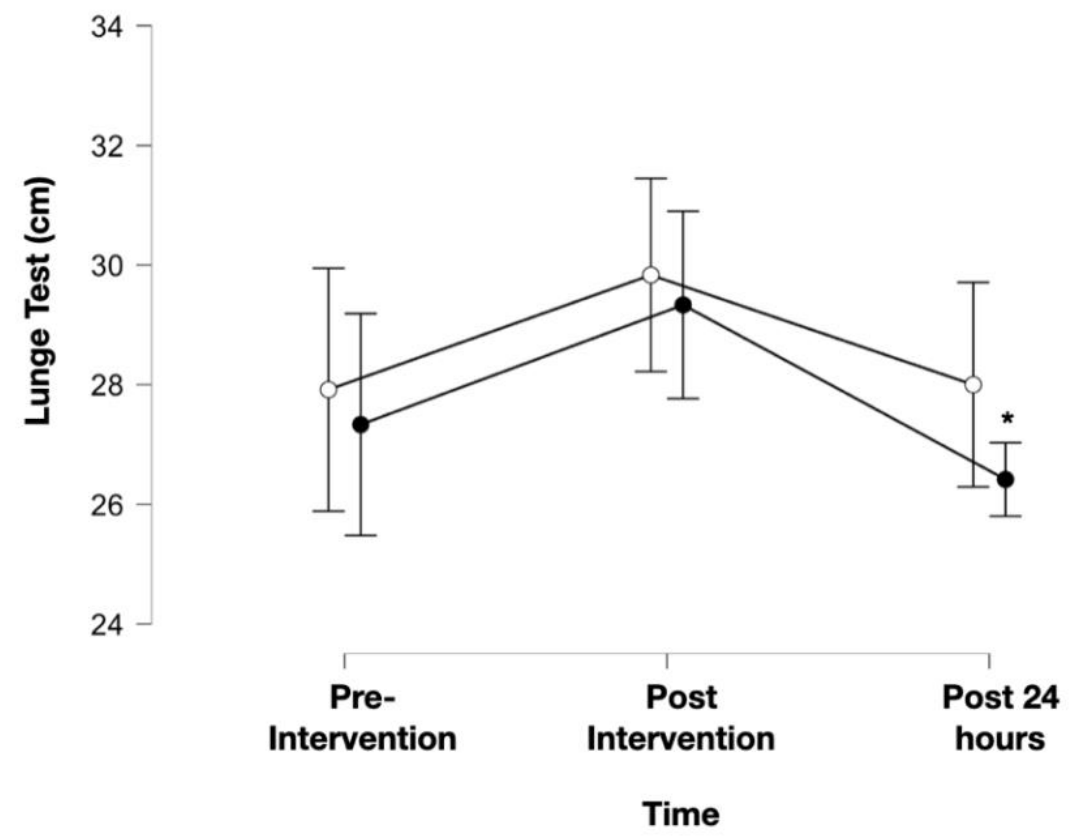

Figure 1. Interaction effect of time (pre, immediate post and $24 \mathrm{~h}$ post intervention) $\mathrm{x}$ intervention (i.e., SFR vs. DN on lunge test score). * Significant differences $(\mathrm{p}=0.049)$ in comparison to post-intervention. 
The RM ANOVA did not show significant differences in main effect of "intervention" variable $\left(\mathrm{F}_{[1,11]}=0.63, \mathrm{p}=0.443\right.$, eta $\left.=0.05\right)$ in hyperalgesia variable. The $\mathrm{MD}, \mathrm{CI} 95 \%$ and ES were -0.35 [-1.33 to 0.62$]$ and 0.23 , respectively. On the other hand, significant differences were found at interaction effect of "time $x$ intervention" $\left(\mathrm{F}_{[2,22]}=4.30, \mathrm{p}=\right.$ $0.027, \mathrm{n}^{2} \mathrm{p}=0.28$ ). Bonferroni post Hoc comparison revealed significant differences in pre intervention vs. post 24 hours post intervention in SFR condition (see figure 2).
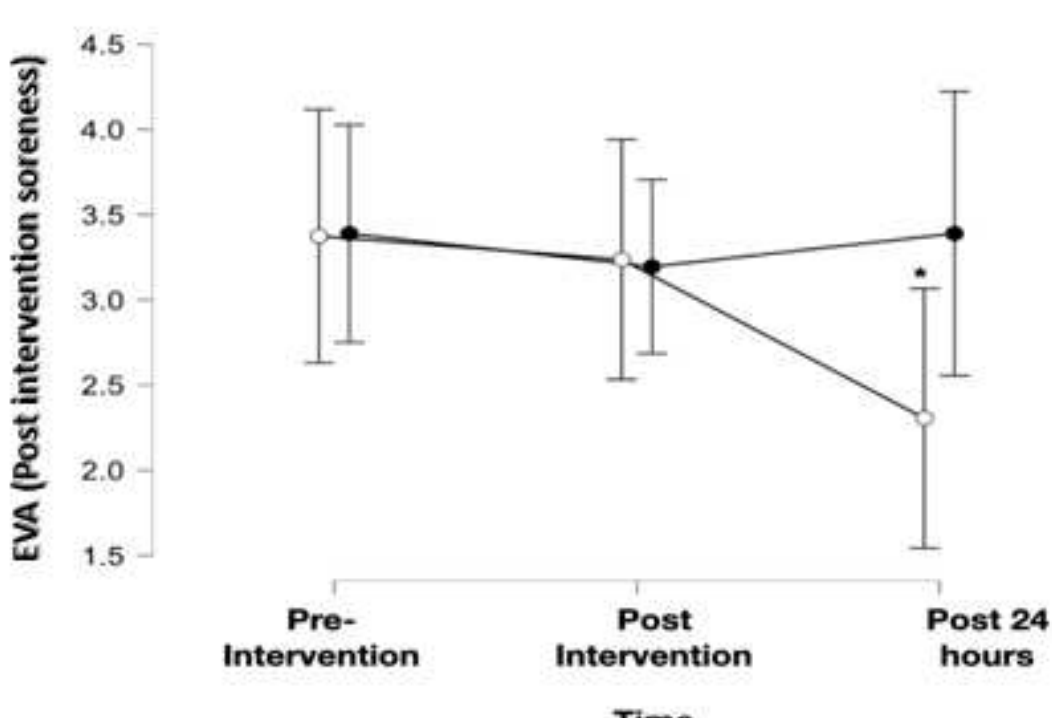

Experimental conditions

Intervención

- Foam

- DryNeedling

Time

Figure 2. Interaction effect of time (pre, immediate post and $24 \mathrm{~h}$ post intervention) $\mathrm{x}$ intervention (i.e., SFR vs. DN) on soreness post interventions. * Significant differences $(\mathrm{p}=0.028)$ in comparison to post-intervention. 
The RM ANOVA did not show significant differences in main effect of "intervention" variable $\left(\mathrm{F}_{[1,11]}=1.23, \mathrm{p}=0.313\right.$, eta $\left.=0.10\right)$ in $\mathrm{CMJ}$ height. The $\mathrm{MD}, \mathrm{CI} 95 \%$ and $\mathrm{ES}$ were -0.54 [ -1.40 to 2.49$]$ and 0.18 , respectively. On the other hand, significant differences were found at interaction effect of "time $x$ intervention" $\left(\mathrm{F}_{[1.24,13.60]}=2.12, \mathrm{p}=0.167, \mathrm{n}^{2} \mathrm{p}\right.$ $=0.16$ ). Bonferroni post Hoc comparison revealed no significant differences (see figure 3.)

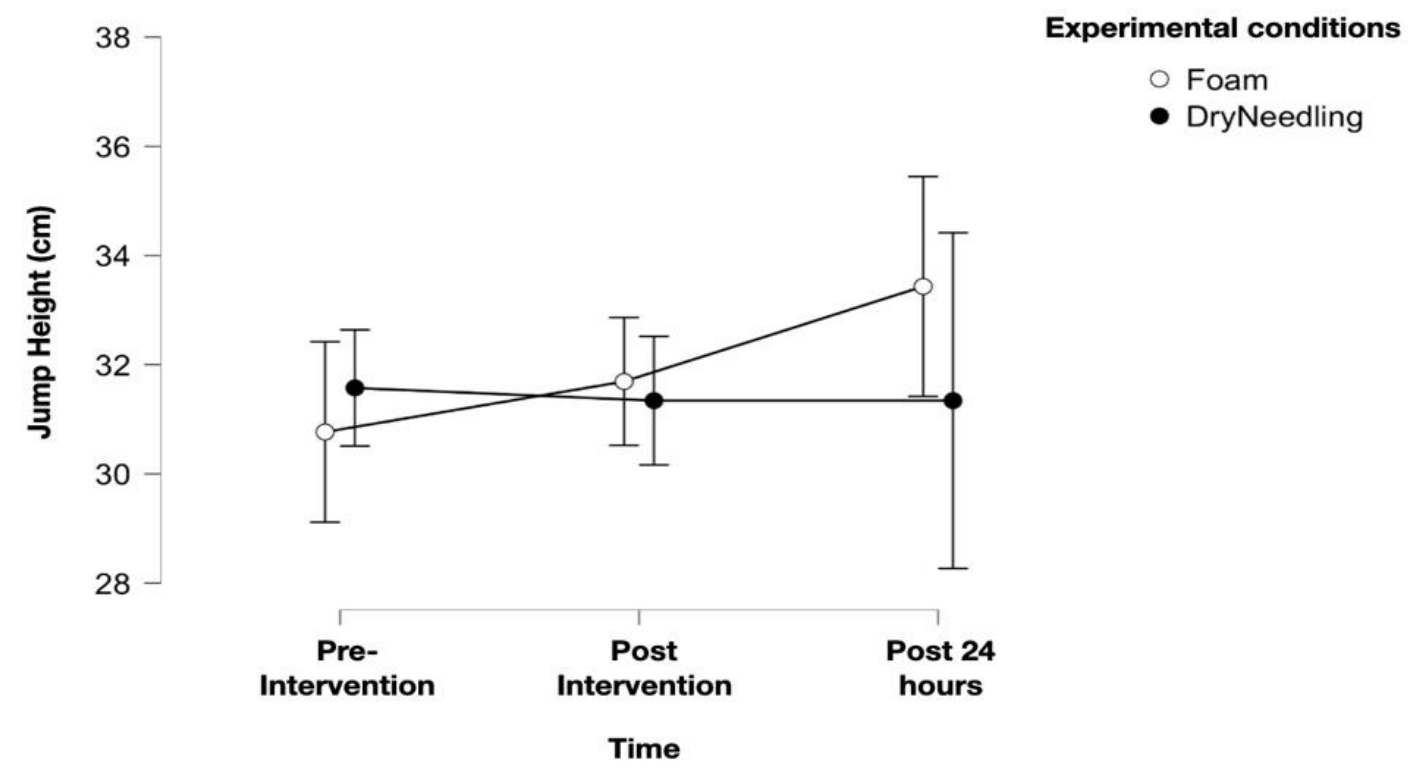

Figure 3. Interaction effect of time (pre, post and $24 \mathrm{~h}$ post intervention) $\mathrm{x}$ intervention (i.e., SFR vs. DN) on jump heigh (cm). Note: bar represent the 95\% CI.

\section{ADVERSE EVENTS}

No adverse events or unintended effects were recorded. 


\section{DISCUSSION}

This study aimed to examine and compare the acute effects of SFR vs. DN techniques on ankle mobility, post-intervention soreness and performance in active and healthy young adults. The main finding of the present study was that both techniques, SFR and DN, reported similar values in all dependent variables assessed (passive ankle dorsiflexion mobility, muscle soreness and jump height). However, although non-statistically significant differences were found between conditions, moderate ES were obtained on post-intervention soreness and jump height in favour of SFR condition at post $24 \mathrm{~h}$ in comparison to DN.

A recent systematic review suggests that both SFR and the roller massage may offer shortterm benefits for increasing sit and reach scores and ROM at the ankle without performance improvements ${ }^{14}$. Our results are partially in line with this current because we found positive changes at immediate post-intervention ankle ROM, but also improvements in jump height at the same moment, although the magnitude of the changes was not enough to be statistically significant. A previous study found that the dorsiflexion ROM immediately increased $6^{\circ}(22 \%)$ in the triceps surae muscle after the SFR intervention ${ }^{15}$. Our results are in accordance with previous studies where the SFR increases the ROM of the ankle joint immediately, both in the athletic and general population ${ }^{16}$. Similarly, Bushell et al reported that SFR induced muscle relaxation and increased ROM in college athletes ${ }^{17}$.

Contrary to some studies, which showed negative effects on performance when SFR was applied in pre-competition ${ }^{18}$, we found that the gains in ankle flexibility did not have a negative impact on performance, evaluated through the jump height in a CMJ. However, 
our results are in line with previous research where knee, ankle, hip and trunk ROM improvements also had no negative consequences on different performance parameters 19. It should be noted that the effects reported in our studies were acute effects. These discrepancies between studies can be related with the heterogeneity in the protocols of SFR applied and in the samples.

Several possibilities such us the activation mechanoreceptors and autonomic nervous system response ${ }^{20}$ could be considered responsible for the changes of the ankle ROM due to the activation of central pain modulatory paths, through neural inhibition mechanisms ${ }^{21}$. In fact, the application to the lower extremity prior to the activity does not enhance or negatively affect muscle performance but may change the perception of fatigue ${ }^{22}$. In fact, there is an emerging consensus on how SFR treatment affects improving power, strength, elasticity and balance in athletes. However, no significant anatomical changes has been observed in fascicle length in the gastrocnemius muscle ${ }^{15}$.

On the other hand, among the existing tools for the treatment of MPS, together with noninvasive myofascial self-liberation techniques such as the aforementioned SFR, there are techniques with an invasive approach such as wet needling or DN. DN has been recommended for management MTrPs pain ${ }^{23}$. MTrPs dry needling procedures have shown to be associated with post-needling soreness which usually lasting less than 72 hours ${ }^{24}$. This post needling soreness will not be of similar intensity in all patients, and some of them may not even perceive said post-intervention sequelae.

Although the symptom is not reported by all patients equally, there are previous studies that show that in asymptomatic patients, the use of DN over latent MTrPs within the 
medial gastrocnemius muscle produced an increase in magnetic resonance imaging using the Short Tau Inversion Recovery sequence (MRI-STIR), compatible with intramuscular edema and an increase of muscle stiffness ${ }^{13}$. Due to this important sideeffect, it is very necessary for physical therapists to know the clinical conditions and time periods for which DN produced post-needling soreness. It is well-known that the number of needle insertions is associated with this symptom ${ }^{25}$. Currently, several strategies has been used to control post-dry needling soreness ${ }^{26}$.

In the case of our study, the SFR not only increases the ROM and improves CMJ height, but also reduced the pain significantly at $24 \mathrm{hrs}$. Previous results suggests there is an acute increase on the PPTs post-foam roller ${ }^{27}$. However, contrary to what could be expected, post-needling soreness was similar in the three evaluation periods (pre, immediate post and $24 \mathrm{~h}$, with no significant increase as a result of the DN intervention. It should be noted that, both SFR and DN conditions achieved similar pre and immediate post muscle soreness, showing only acute and significant effects between interventions at 24 hours in favor of SFR but due to a decrease in the VAS values of the SFR, not to an increase as a consequence of the DN intervention.

It is important to highlight that, corroborating previous studies where the use of SRF was not associated with a decrease in strength and performance after its immediate application ${ }^{28}$, the use of DN seems to follow the same behavior after treatment on the variables studied in the present investigation. It seems, therefore, that, despite the tissue damage generated by $\mathrm{DN}$, both techniques can be used to increase ankle dorsiflexion mobility and performance without an increase in magnitude and duration of acute post-treatment pain. In addition, no adverse events were recorded during the study period. 
Our study is not without limitations, which must be considered. Firstly, there is no significant differences between conditions, but it is possible to appreciate that there is a tendency in the post-intervention soreness in favour of SFR, being the small sample size used in the study (type I error) possible bias. Secondly, the lack of measures (i.e., ultrasound) to identify possible structural (intra-articular) artifacts associated with dorsiflexion limitation. Lastly, the absence of objective diagnosis for the detection of MTrPs with the use of ultrasound.

Finally, despite the widespread use of SFR and DN in sport and clinic fields, the current literature measuring the effects of SFR and DR as a preventive, treatment or recovery performance tools is still emerging. Thus, futures studies should investigate the effects of these techniques using different protocols and samples, and implemented short and longterm interventions to analyze their chronic effects.

\section{METHODS}

\section{Experimental approach to the problem}

A randomized prospective crossover trial design was used to compare the acute effects of SFR and interventions in terms of magnitude and duration on the physiological variables of ankle mobility (ROM), pain (PPTs and visual analogue scale; VAS) and performance (countermovement $[\mathrm{CMJ}]$ height) in active and healthy young adults. The conditions of SFR and DN were randomized and counterbalanced among participants to control the sequential effect of the order and transfer through by an independent investigator using Epidat 3.1 (www.sergas.es). The author V-M J. implemented the allocation sequence, while B-V J. enrolled the participants and assigned participants to interventions. All 
dependent variables were measured at pre-intervention, immediate post-intervention and post-intervention at $24 \mathrm{~h}$ (Figure 4). Data collectors and data analytics were blinded to interventions.

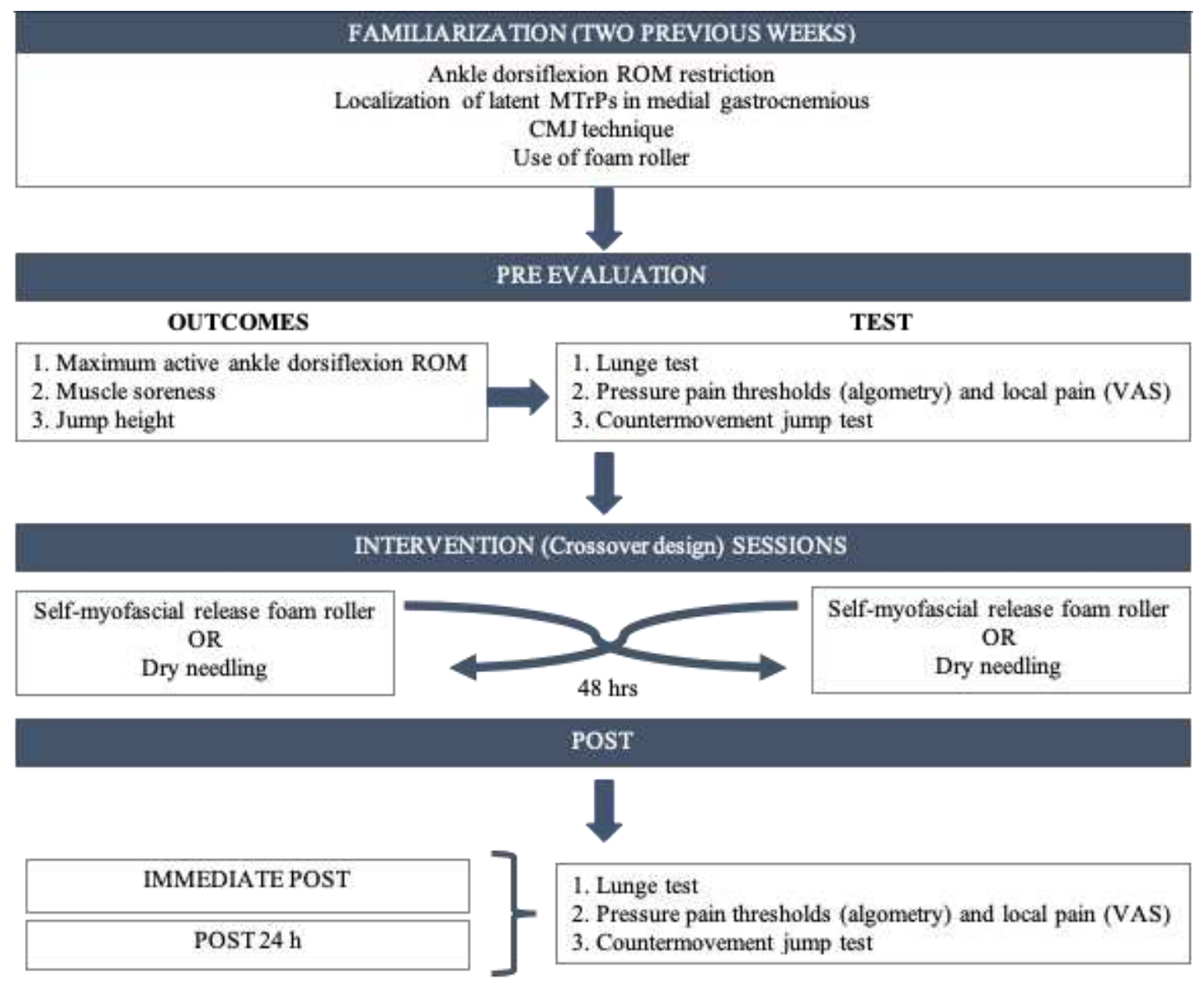

ROM: range of motion; MTrPS: myofascial trigger points; CMJ: countermovement jump; VAS: visual analog scale

Figure 4. Intervention design

\section{Ethics}

The study adhered to the CONSORT guidelines ${ }^{29}$. It was conducted in accordance with the Declaration of Helsinki and approval by the local ethics committee of the Catholic University of Valencia was obtained (UCV/2019-2020/062). Each included subject signed informed consent. The trial was registered $(1 / 05 / 2020)$ at clinicaltrials.gov (NCT04600830).

\section{Subjects}


A total of 12 participants were involved in the present study. The sample was collected through an informative form for participation in a research study. The participants were young healthy and active male adults (age: $23.41 \pm 1.68$ years; height: $1.79 \pm 0.088 \mathrm{~m}$; body mass: $78.33 \pm 9.03 \mathrm{Kg}$; body mass index (BMI): $24.14 \pm 1.45 \mathrm{~kg} / \mathrm{m}^{2}$ ). Participants were students recruited from the Catholic University of Valencia through initial screening to select the participants who met the following inclusion criteria: active ankle dorsiflexion restriction $(<11.5 \mathrm{~cm} \text { or } 1.5 \mathrm{~cm} \text { difference between both limbs })^{30}$. Exclusion criteria were as follow: (i) chronic pain syndromes such as low back pain, fibromyalgia or others, (ii) skin injuries in the area to be treated, (iii) peripheral and central neuropathic pain, (iv) recent surgery in the lower limbs (last 12 months), (v) cardiovascular, neurological, inflammatory and rheumatic diseases; and (vi) engage in physical activity/sports during the total length of the experimental study.

\section{Procedures}

The study encompassed three sessions. During the first session (familiarization) performed two prior weeks, the ankle dorsiflexion ROM was assessed to identify the subjects with mobility restriction during this movement (regardless of whether the restriction was present in the dominant or non-dominant limb.) During this session, with the participants finally included, latent MTrPs in medial gastrocnemius muscle were screened following the International Delphi instructions ${ }^{31}$. Aiming to reduce locationspecific variance between participants and assessments, the test were performed by a trained staff (physiotherapist) in the area of MPS and trigger points according to Travell and Simons procedures ${ }^{32}$. The gastrocnemius muscle was chosen because it has been shown to exhibit the highest prevalence of latent MTrPs in healthy subjects ${ }^{33}$. Furthermore, the first session was used to familiarize the sample with the use of the foam roller and the CMJ procedure (explanation and performing the technique four times). 
During the second session, the interventions techniques along with the assessments of the dependent variables were carried out. The order of the assessments was: lunge test, CMJ height and post-intervention pain through PPTs and VAS. Both sessions (50 min each one) were performed in university clinics during the last week of May with a rest period of 48 hours between sessions. Participants were also instructed to refrain from taking any medications that would interfere with testing and maintain their usual nutrition intake and physical activity levels.

\section{Intervention}

\section{$\underline{\text { Foam Roller }}$}

The myofascial self-release technique with the uniform polystyrene Black Roll PRO foam roller (Bottighogen, Switzerland; density $2.3 \mathrm{Kg} / \mathrm{m}^{3} ; 15 \times 91 \mathrm{~cm}$ [diameter x length]) was performed on the ground with the participant lying over the foam roller. The foam roller was placed under the gastro-soleus complex, while the subject slowly moved his body in the same direction as the muscle fibers, using his hands to push himself and make the roller slide back and forth. The intervention divided the gastrocnemius into two zones. The zone one and two corresponded to the medial and lateral gastrocnemius, respectively.

The device was only applied at the muscular level, avoiding the Achilles tendon area and the popliteal fossa ${ }^{15}$. A total of three sets of 60 -sec with 30 -sec rest between them were performed on the lower limb with the ankle mobility restriction. The subjects performed around 30 self-myofascial release cycles in the target area during the minute $(\sim 1$ second each back and forward movement measured with metronome) (Smart Metronome; Tomohiro Ihara, Japan) ${ }^{34}$. In detail the subjects completed a total of 90 cycles in three minutes ( 3 sets x 30 cycles each set). A research member recorded the cycles. This total 
time was stablished based on the lack of effects on ROM when SFR is applied during two minutes ${ }^{35}$. The pressure intensity was the maximum tolerable. Pressure was adjusted by applying body weight to the roller and using the hands and feet to offset weight as required. The subjects underwent prior familiarization before completing the intervention and were instructed at all times. Each participant practiced 3 or 4 times to learn the correct technique with the supervision of a research staff. Each participant performed warm-up exercises (5 minutes of light aerobic cycling at $60 \%$ of maximum heart rate was initiated) before all participants performed the pre-test.

\section{Dry Needling}

One session of DN were carried out. DN were performed with disposable stainless Steel needles ( $0,3 \times 40 \mathrm{~mm}$. Myofib, Toledo, Spain) and applied manually by the therapists ( $>10$ years of experience) on the latent MTrPs of medial gastrocnemius area. The area was cleaned previously by the therapist with alcohol and always sterile gloves were used. Hong's fast-in and fast-out technique was implemented during the DN intervention ${ }^{36}$. The needle was moved up and down 2-3 mm vertically through the latent MTrPS, which was located within the a muscle taut band ${ }^{34}$. Approximately 25 insertions without leaving the skin during half minute were implemented ${ }^{32}$. At the end of the intervention, a cotton piece was applied to the treated area for one minute to ensure an adequate control of hemostasis.

\section{Parameters}

\section{Post-intervention muscle soreness}

Pressure pain sensibility (primary outocome), named also as pressure pain perception, is defined as the minimal amount of pressure at which the sense of pressure first changes to pain ${ }^{37}$. The PPT was measured with mechanical stimuli which were applied using a 
manual analogue algometer with a Wagner FDK/FDN series Force Dial analogue Fisher algometer (Wagner Instruments, Greenwich, CT). Firstly, the subjects were familiarized with the procedure ${ }^{38}$. It was determined by deep palpation of MTrPs location on the interventional sides of medial gastrocnemius (trigger point 2). After identification of a taut band, a hard hubber probe $\left(1 \mathrm{~cm}^{2}\right)$ was placed perpendicular to the skin ${ }^{39}$. The examiner applied increasing pressure up $4 \mathrm{~kg} / \mathrm{cm}^{2}$ was applied with the thumb to the most sensitive tender spot/nodule for 5 seconds, and the subject had of pain using a $0-10 \mathrm{~cm}$ VAS ruler with 2 extremes: no pain and worst pain ever felt ${ }^{40}$.

The position used is shown in Figure 5. The PPTs was measured three times. The mean value from the 3 assessments was used in the analyses ${ }^{41}$. PPTs was assessed before and immediately after the needling procedure, and at 24 hours after intervention. Visual analogue scale this procedure has demonstrated good reliability ${ }^{42}$.

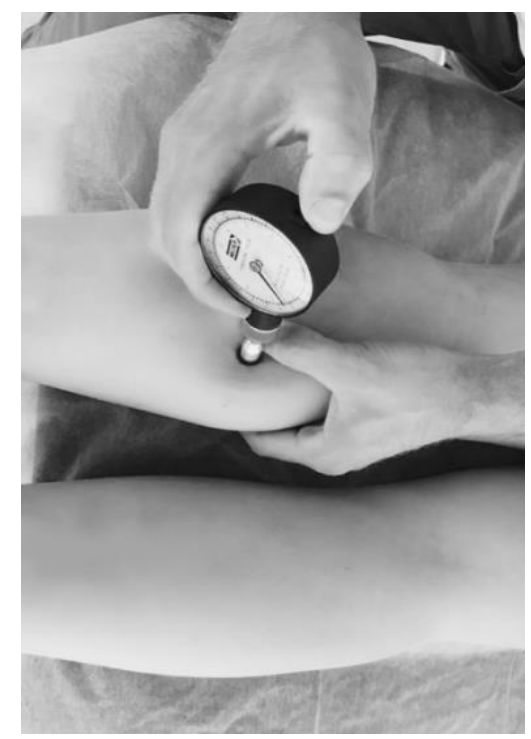

Figure 5. Measurement of the pressure pain threshold at the MTrPs treated. 


\section{Weight-bearing lunge test}

To evaluate ankle mobility, the weight-bearing lunge test was used. The participants lunged forward trying to touch a vertical line on the wall with their knee while maintaining the heel of the same limb evaluated in contact with the ground. They moved their foot away from the wall in order to reach maximum dorsiflexion $\mathrm{ROM}^{43}$. The foot of each participant was positioned so that the horizontal line on the base of the platform was between the 2 nd and 3 rd toes and bisected the calcaneus ${ }^{44}$. Participants were instructed to lunge their knee forward, keeping their heel in contact with the platform. The subjects performed three familiarization trials followed by three test trials without rest interval between these two phases and between trials. The mean distance in $\mathrm{cm}$ of the three trials was recorded. During the test, the subjects were allowed to put the non-tested leg in a comfortable position behind the testing limb and to hold onto the wall to maintain their balance ${ }^{45}$. Participants were instructed to blend the ankle and knee as far as possible, but no encouragement was provided during the testing.

\section{Countermovement jump}

The CMJ jump height was measured with a light barrier system (OptoGait, Microgate, Bolzano Bozen, Italy) which has been used in similar studies ${ }^{46}$. This device measures the contact time on the floor and the flight time, using photoelectric cells. Participants were placed standing with each foot on the floor (barefoot) at shoulder height and with hands akimbo, then, restricting arm movements to be focused only on the force generated via lower limbs ${ }^{47}$. Participants were instructed to lower themselves as low as possible, as 
quickly as possible and jump as high as possible vertically, returning to the standing position after landing. They performed three attempts with 15 seconds rest between them, as has been established in previous studies. The research staff team control visually the correct technique of each attempt to identify any possible mistake despite all participants performed a previous familiarization session where learned the correct execution (at least four jumps, two weeks before). Flight time was used to calculate the height of the rise using the body's centre of gravity ${ }^{48}$. The typically observed warm-up consisted of squatting movements, toe touches, hopping, and practice jumps, and lasted 3-5 minutes 49. The average value of the three attempts was used for the subsequent analysis.

\section{Statical Analysis}

All variables were expressed as a mean and standard deviation (SD). The assumption of normality was assessed using Kolmogorov-Smirnov ( $p>0.05$ ). Repeated measures analysis of variance (ANOVA RM) was used to determine the effect of intervention technique (i.e., foam roller or dry needling) in ankle dorsiflexion ROM, muscle soreness and jump height. All comparisons between measures were corrected using Mauchly test (i.e., Greenhouse-Geisser approximation). For all analyses, when a significant main effect was detected, post hoc related Student's t-test with Bonferroni corrections was used to determine which comparisons differed. The Cohen effect size (ES) used was expressed as the difference of typified mean change. The ES was considered trivial $(<0.20)$, small $(0.20-0.59)$, moderate $(0.60-1.19)$, large $(1.20-1.99)$ and very large $(>2.00)$. The Pearson correlation coefficient was used to analyse the association between variables. A "trivial" association was established as $\mathrm{r}<0.25$, "small" between $0.25<\mathrm{R}<0.50$, "moderate to strong" between $0.50<\mathrm{r}<0.75$ and "very strong" as $r>0.75$. The significance level was set at $\mathrm{p}<0.05$. All analyses were performed using statistical 
analysis software (SPSS Inc, Chicago, Illinois, USA). Sample size was calculated based on the effect size reported by Sánchez-Infante et al. (2021) who measured "pressure pain perception" (ES $=0.61)$, considering the probability of loss during follow-up (30\%) the

minimal number of participants, the minimal required to attain a power of 0.9 and a bilateral $\alpha$ level of 0.05 for analysis of variance (ANOVA) with repeated measures.

\section{CONCLUSIONS}

Both SFR and DN are effective to improve ankle dorsiflexion and performance in healthy young male adults without generating an acute negative effect in muscle soreness. Then, both types of strategies could be used since the pain generated by both tools does not affect the performance and ankle dorsiflexion, although future studies with higher sample size and with different population are needed.

\section{ACKNOWLEDGMENTS}

We would like to thanks to all participants of the present study for their time.

\section{AUTHOR CONTRIBUTIONS STATEMENT}

V-M J and B-V L conceived and designed the experiment; L-S J, T-V J and GP conducted the experiment and collected the data; B IJ performed the statistical analysis. All authors wrote and reviewed the manuscript.

\section{FUNDING}

This research did not receive any funding from agencies in the public, private, or not-forprofit sectors. 


\section{COMPETING INTEREST}

The authors of the present manuscript declare no competing interests.

\section{DATA AVAILABILITY STATEMENT}

The datasets generated during and/or analysed during the current study are available from the corresponding author on reasonable request.

\section{REFERENCES}

1. Weller, J. L., Comeau, D. \& Otis, J. A. Myofascial pain. in vol. 38 640-643 (Thieme Medical Publishers, 2018).

2. Bron, C. \& Dommerholt, J. D. Etiology of myofascial trigger points. Curr. Pain Headache Rep. 16, 439-444 (2012).

3. Barbero, M., Schneebeli, A., Koetsier, E. \& Maino, P. Myofascial pain syndrome and trigger points: evaluation and treatment in patients with musculoskeletal pain. Curr. Opin. Support. Palliat. Care 13, 270-276 (2019).

4. Simons, D. G., Travell, J. \& Simons, L. S. Myofascial pain and dysfunction: the trigger point manual: volume 1. (1999).

5. Skinner, B., Moss, R. \& Hammond, L. A systematic review and meta-analysis of the effects of foam rolling on range of motion, recovery and markers of athletic performance. J. Bodyw. Mov. Ther. 24, 105-122 (2020).

6. Skinner, B., Moss, R. \& Hammond, L. A systematic review and meta-analysis of the effects of foam rolling on range of motion, recovery and markers of athletic performance. J. Bodyw. Mov. Ther. 24, 105-122 (2020). 
7. Hendricks, S., den Hollander, S., Lombard, W. \& Parker, R. Effects of foam rolling on performance and recovery: A systematic review of the literature to guide practitioners on the use of foam rolling. J. Bodyw. Mov. Ther. 24, 151-174 (2020).

8. Espejo-Antúnez, L. et al. Dry needling in the management of myofascial trigger points: a systematic review of randomized controlled trials. Complement. Ther. Med. 33, 46-57 (2017).

9. Gattie, E., Cleland, J. A. \& Snodgrass, S. The effectiveness of trigger point dry needling for musculoskeletal conditions by physical therapists: a systematic review and meta-analysis. J. Orthop. Sports Phys. Ther. 47, 133-149 (2017).

10. Wiewelhove, T. et al. A Meta-Analysis of the Effects of Foam Rolling on Performance and Recovery. Front. Physiol. 10, 376 (2019).

11. Wiewelhove, T. et al. A meta-analysis of the effects of foam rolling on performance and recovery. Front. Physiol. 10, 376 (2019).

12. Martín-Pintado-Zugasti, A., Del Moral, O. M., Gerwin, R. D. \& Fernández-Carnero, J. Post-needling soreness after myofascial trigger point dry needling: Current status and future research. J. Bodyw. Mov. Ther. 22, 941-946 (2018).

13. Baraja-Vegas, L. et al. Localization of muscle edema and changes on muscle contractility after dry needling of latent trigger points in the gastrocnemius muscle. Pain Med. 20, 1387-1394 (2019).

14. Cheatham, S. W., Kolber, M. J., Cain, M. \& Lee, M. The effects of self-myofascial release using a foam roll or roller massager on joint range of motion, muscle recovery, and performance: a systematic review. Int. J. Sports Phys. Ther. 10, 827$838(2015)$. 
15. Yoshimura, A. et al. Effects of Self-myofascial Release Using a Foam Roller on Range of Motion and Morphological Changes in Muscle: A Crossover Study. J. Strength Cond. Res. (2019).

16. Bush, H. M., Stanek, J. M., Wooldridge, J. D., Stephens, S. L. \& Barrack, J. S. Comparison of the Graston Technique ${ }^{\circledR}$ With Instrument-Assisted Soft Tissue Mobilization for Increasing Dorsiflexion Range of Motion. J. Sport Rehabil. 1, 1-8 (2020).

17. Bushell, J. E., Dawson, S. M. \& Webster, M. M. Clinical relevance of foam rolling on hip extension angle in a functional lunge position. J. Strength Cond. Res. 29, $2397-2403$ (2015).

18. Phillips, J., Diggin, D., King, D. L. \& Sforzo, G. A. Effect of varying selfmyofascial release duration on subsequent athletic performance. J. Strength Cond. Res. 35, 746-753 (2021).

19. Richman, E. D., Tyo, B. M. \& Nicks, C. R. Combined Effects of Self-Myofascial Release and Dynamic Stretching on Range of Motion, Jump, Sprint, and Agility Performance. J. Strength Cond. Res. 33, 1795-1803 (2019).

20. Schleip, R. Fascial plasticity-a new neurobiological explanation Part 2. J. Bodyw. Mov. Ther. 7, 104-116 (2003).

21. Cavanaugh, M. T. et al. An acute session of roller massage prolongs voluntary torque development and diminishes evoked pain. Eur. J. Appl. Physiol. 117, 109$117(2017)$

22. Peacock, C. A. et al. Comparing acute bouts of sagittal plane progression foam rolling vs. frontal plane progression foam rolling. J. Strength Cond. Res. 29, 2310$2315(2015)$ 
23. Navarro-Santana, M. J. et al. Effectiveness of Dry Needling for Myofascial Trigger Points Associated with Neck Pain Symptoms: An Updated Systematic Review and Meta-Analysis. J. Clin. Med. 9, 3300 (2020).

24. Martín-Pintado-Zugasti, A., Del Moral, O. M., Gerwin, R. D. \& Fernández-Carnero, J. Post-needling soreness after myofascial trigger point dry needling: Current status and future research. J. Bodyw. Mov. Ther. 22, 941-946 (2018).

25. Martín-Pintado-Zugasti, A. et al. Postneedling Soreness and Tenderness After Different Dosages of Dry Needling of an Active Myofascial Trigger Point in Patients With Neck Pain: A Randomized Controlled Trial. PM R 10, 1311-1320 (2018).

26. Martín-Pintado-Zugasti, A. et al. Ischemic Compression After Dry Needling of a Latent Myofascial Trigger Point Reduces Postneedling Soreness Intensity and Duration. PM R 7, 1026-1034 (2015).

27. Vaughan, B., McLaughlin, P. \& Lepley, A. S. Immediate changes in pressure pain threshold in the iliotibial band using a myofascial (foam) roller. Int. J. Ther. Rehabil. 21, 569-574 (2014).

28. Kalichman, L. \& David, C. B. Effect of self-myofascial release on myofascial pain, muscle flexibility, and strength: a narrative review. J. Bodyw. Mov. Ther. 21, 446451 (2017).

29. Grant, S. et al. CONSORT-SPI 2018 Explanation and Elaboration: guidance for reporting social and psychological intervention trials. Trials 19, 406 (2018).

30. Hoch, M. C. \& McKeon, P. O. Normative range of weight-bearing lunge test performance asymmetry in healthy adults. Man. Ther. 16, 516 (2011). 
31. Fernández-de-las-Peñas, C. \& Dommerholt, J. International Consensus on Diagnostic Criteria and Clinical Considerations of Myofascial Trigger Points: A Delphi Study. Pain Med. 19, 142-150 (2018).

32. Dommerholt, J. \& de las Penas, C. F. Trigger Point Dry Needling E-Book: An Evidence and Clinical-Based Approach. (Elsevier Health Sciences, 2018).

33. Wilke, J., Vogt, L. \& Banzer, W. Immediate effects of self-myofascial release on latent trigger point sensitivity: a randomized, placebo-controlled trial. Biol. Sport 35, 349 (2018).

34. Moral, O. M. del. Dry needling treatments for myofascial trigger points. $J$. Musculoskelet. Pain 18, 411-416 (2010).

35. Couture, G., Karlik, D., Glass, S. C. \& Hatzel, B. M. The effect of foam rolling duration on hamstring range of motion. Open Orthop. J. 9, 450 (2015).

36. Núñez-Cortés, R., Cruz-Montecinos, C., Vásquez-Rosel, Á., Paredes-Molina, O. \& Cuesta-Vargas, A. Dry Needling Combined With Physical Therapy in Patients With Chronic Postsurgical Pain Following Total Knee Arthroplasty: A Case Series. J. Orthop. Sports Phys. Ther. 47, 209-216 (2017).

37. Vanderweeën, L., Oostendorp, R. A. B., Vaes, P. \& Duquet, W. Pressure algometry in manual therapy. Man. Ther. 1, 258-265 (1996).

38. Kamali, F., Sinaei, E. \& Morovati, M. Comparison of upper trapezius and infraspinatus myofascial trigger point therapy by dry needling in overhead athletes with unilateral shoulder impingement syndrome. J. Sport Rehabil. 28, 243-249 (2019).

39. Cudejko, T. et al. Decreased pain and improved dynamic knee instability mediate the beneficial effect of wearing a soft knee brace on activity limitations in patients with knee osteoarthritis. Arthritis Care Res. 71, 1036-1043 (2019). 
40. Sánchez-Infante, J., Bravo-Sánchez, A., Jiménez, F. \& Abián-Vicén, J. Effects of dry needling on mechanical and contractile properties of the upper trapezius with latent myofascial trigger points: A randomized controlled trial. Musculoskelet. Sci. Pract. 56, 102456 (2021).

41. Steen Pettersen, P. et al. Peripheral and central sensitization of pain in individuals with hand osteoarthritis and associations with Self-Reported pain severity. Arthritis Rheumatol. 71, 1070-1077 (2019).

42. Oliveira-Campelo, N. M., de Melo, C. A., Alburquerque-Sendín, F. \& Machado, J. P. Short-and medium-term effects of manual therapy on cervical active range of motion and pressure pain sensitivity in latent myofascial pain of the upper trapezius muscle: a randomized controlled trial. J. Manipulative Physiol. Ther. 36, 300-309 (2013).

43. Hall, E. A. \& Docherty, C. L. Validity of clinical outcome measures to evaluate ankle range of motion during the weight-bearing lunge test. J. Sci. Med. Sport 20, 618-621 (2017)

44. Smith, M. D. et al. How much does the talocrural joint contribute to ankle dorsiflexion range of motion during the weight-bearing lunge test? A cross-sectional radiographic validity study. J. Orthop. Sports Phys. Ther. 49, 934-941 (2019).

45. Langarika-Rocafort, A., Emparanza, J. I., Aramendi, J. F., Castellano, J. \& CallejaGonzález, J. Intra-rater reliability and agreement of various methods of measurement to assess dorsiflexion in the Weight Bearing Dorsiflexion Lunge Test (WBLT) among female athletes. Phys. Ther. Sport 23, 37-44 (2017).

46. Lehance, C., Binet, J., Bury, T. \& Croisier, J.-L. Muscular strength, functional performances and injury risk in professional and junior elite soccer players. Scand. J. Med. Sci. Sports 19, 243-251 (2009). 
47. Lees, A., Vanrenterghem, J. \& De Clercq, D. Understanding how an arm swing enhances performance in the vertical jump. J. Biomech. 37, 1929-1940 (2004).

48. García-Pinillos, F., Ruiz-Ariza, A., Moreno del Castillo, R. \& Latorre-Román, P. Impact of limited hamstring flexibility on vertical jump, kicking speed, sprint, and agility in young football players. J. Sports Sci. 33, 1293-1297 (2015).

49. Barker, L. A., Harry, J. R. \& Mercer, J. A. Relationships between countermovement jump ground reaction forces and jump height, reactive strength index, and jump time. J. Strength Cond. Res. 32, 248-254 (2018). 